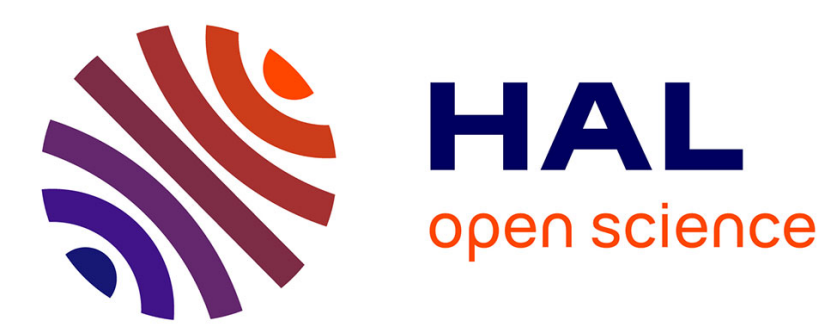

\title{
Ajustement des données d'insolation d'Alger par un modèle Markovien du premier ordre
}

\author{
A. Maafi, A. Adane, A. Ouabdesselam
}

\section{To cite this version:}

A. Maafi, A. Adane, A. Ouabdesselam. Ajustement des données d'insolation d'Alger par un modèle Markovien du premier ordre. Revue de Physique Appliquée, 1987, 22 (6), pp.425-430. 10.1051/rphysap:01987002206042500 . jpa-00245557

\section{HAL Id: jpa-00245557 https://hal.science/jpa-00245557}

Submitted on 1 Jan 1987

HAL is a multi-disciplinary open access archive for the deposit and dissemination of scientific research documents, whether they are published or not. The documents may come from teaching and research institutions in France or abroad, or from public or private research centers.
L'archive ouverte pluridisciplinaire HAL, est destinée au dépôt et à la diffusion de documents scientifiques de niveau recherche, publiés ou non, émanant des établissements d'enseignement et de recherche français ou étrangers, des laboratoires publics ou privés. 


\title{
Ajustement des données d'insolation d'Alger par un modèle Markovien du premier ordre
}

\author{
A. Maafi $(*)$, A. Adane $\left({ }^{* *}\right)$ et A. Ouabdesselam (*) \\ Ecole Nationale Polytechnique, Département d'Electronique, Avenue Pasteur, El Harrach (Alger), Algérie
}

(Reçu le 2 juin 1986, révisé le 2 février 1987, accepté le 3 mars 1987)

\begin{abstract}
Résumé. - Les données journalières d'insolation enregistrées à Alger de 1972 à 1982, ont fait l'objet d'une analyse statistique. Par comparaison des fractions d'insolation $S S / S S_{0}$ à une suite de valeurs de seuil $S_{\mathrm{g}}$, on trouve que le rayonnement solaire d'Alger peut être décrit par un processus de Markov du premier ordre à deux états. Le meilleur ajustement de ce modèle est obtenu pour un seuil global $\bar{S}_{\mathrm{g}_{0}}$ égal à 0,43 .

Abstract. - The daily insolation data recorded at Algiers from 1972 to 1982, have been statistically analysed. In comparing the insolation ratios $S S / S S_{\mathrm{o}}$, to a sequence of threshold values $S_{\mathrm{g}}$, it is found that the solar radiation of Algiers can be well described by a first-order two state Markovian process. The best fit of this model is obtained when the global threshold $\bar{S}_{\mathrm{g}_{0}}$ is equal to 0.43 .
\end{abstract}

\section{Introduction.}

La conversion de l'énergie solaire sous une forme adaptée à nos besoins en énergie pose le problème de la modélisation du rayonnement solaire.

En effet, l'optimisation des dimensions des systèmes solaires nécessite une évaluation correcte des paramètres utiles du flux d'énergie solaire. Une bonne approche de calcul de la taille d'un générateur solaire et de ses organes de stockage consiste à élaborer un modèle probabiliste qui tienne compte de deux états du climat : le beau temps et le mauvais temps. Un certain nombre d'études [1, 2], basées pour la plupart sur des modèles stochastiques ou sur les travaux de modélisation des séries temporelles de Box et Jenkins [3], ont été développées dans ce sens. Mais, elles ont été surtout appliquées soit à la prédiction météorologique [1], soit à la conversion thermodynamique de l'énergie solaire [2].

Dans le cas de la conversion photovoltaïque, la plupart des utilisateurs se basent sur des modèles simplifiés de l'irradiation solaire pour déterminer le nombre de panneaux solaires et la capacité des

(*) Ecole Nationale Polytechnique.

(**) Institut d'Electronique, U.S.T.H.B. organes de stockage [4]. Mais avec de tels modèles, les problèmes de stockage d'énergie demeurent mal résolus. En particulier, les approximations mises en jeu dans ces modèles font qu'on ne sait pas encore bien minimiser le rapport coût/performance. On peut alors se demander si un modèle d'irradiation solaire, élaboré à partir de processus stochastiques, peut être appliqué à la conversion photovoltaïque et conduire à de meilleurs résultats.

Les premières études du caractère stochastique du rayonnement solaire sont dues à $\mathrm{B}$. J. Brinkworth [5]. Il a trouvé que les caractéristiques séquentielles des données journalières d'irradiation globale, mesurées en Grande-Bretagne, peuvent être correctement représentées à l'aide d'un modèle autorégressif du premier ordre. Le même type de résultat a été obtenu par U. Amato et al. en Italie [6]. L'étude des systèmes thermodynamiques faite par $\mathbf{R}$. Lestienne [7] a montré que les données d'insolation du Sud de la France pouvaient être également décrites à l'aide d'un modèle en chaîne de Markov du premier ordre.

Dans cet article, nous présentons le modèle en chaîne de Markov du premier ordre à deux états que nous avons mis au point à partir des données d'ensoleillement d'Alger. Ces données ont été mesurées au voisinage de l'Aéroport d'Alger sur une période allant de 1972 à 1982 . 
2. Modélisation du rayonnement solaire par un processus de Markov.

2.1 Formulation GÉNÉrale. - Soient $\phi_{1}$ l'état de beau temps et $\phi_{0}$ l'état de mauvais temps. La figure 1 montre que les transitions possibles entre ces deux états sont au nombre de quatre. Appelons $X_{j}$ la variable aléatoire qui prend l'une des valeurs 1 ou 0 selon qu'au $j$-ième jour le temps soit beau ou mauvais. Dans le cas d'une chaîne de Markov du premier ordre [8], la matrice de transition est par définition :

$$
p=\left[\begin{array}{ll}
p_{11} & p_{10} \\
p_{01} & p_{00}
\end{array}\right]
$$

avec :

$$
\begin{aligned}
& p_{11}=P\left(X_{j+1}=1 / X_{j}=1\right) \\
& p_{10}=P\left(X_{j+1}=0 / X_{j}=1\right) \\
& p_{01}=P\left(X_{j+1}=1 / X_{j}=0\right) \\
& p_{00}=P\left(X_{j+1}=0 / X_{j}=0\right) \\
& p_{10}=1-p_{11} \\
& p_{01}=1-p_{00} .
\end{aligned}
$$

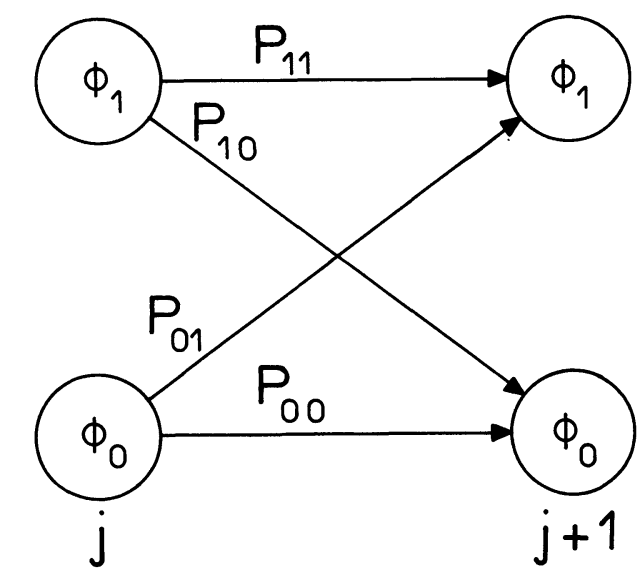

Fig. 1. - Transitions possibles entre les deux états $\phi_{1}$ et $\phi_{0}$.

2.2 LA MÉTHOde D'AJUSTEMENT. - Les données que nous avons utilisées, ont été enregistrées à la Station Météorologique de Dar El Beida (Alger) pendant la période 1972/82. Elles comprennent essentiellement des mesures journalières d'insolation et d'irradiation globale, effectuées respectivement à l'aide d'un héliographe de Campbell et d'un solarimètre horizontal. Celles-ci ont fait l'objet d'un traitement sur ordinateur et d'une analyse statistique approfondie, effectuée mois par mois cumulés sur onze ans.

Au préalable, chacune des durées d'ensoleillement $S S$ a été divisée par la durée astronomique $S S_{\mathrm{o}}=$ $\frac{2}{15} \operatorname{Arc} \cos (-\tan L \cdot \tan D)$ (où $L$ est la latitude du lieu et $D$, la déclinaison). Cette opération permet de soustraire les données d'insolation à l'influence des variations saisonnières. Les fractions d'insolation ainsi obtenues sont ensuite comparées à une valeur de référence $S_{\mathrm{g}}$, comprise entre 0 et 1 , appelée seuil de fraction d'insolation. Celui-ci divise la totalité des données $S S / S S_{\mathrm{o}}$ en deux sous-ensembles. L'un regroupe les valeurs $\frac{S S}{S S_{\mathrm{o}}} \geqslant S_{\mathrm{g}}$ et l'autre se compose de toutes les données $\frac{S S}{S S_{\mathrm{o}}}<S_{\mathrm{g}}$. Le système météorologique associé à ces fractions d'insolation, comporte donc deux états : $\Phi_{1}$ et $\Phi_{0}$. L'état $\Phi_{1}$ correspond à $\frac{S S}{S S_{\mathrm{o}}} \geqslant S_{\mathrm{g}}$. Par contre, le système se trouve à l'état $\Phi_{0}$ si $\frac{S S}{S S_{\mathrm{o}}}<S_{\mathrm{g}}$

Quand on se fixe le seuil dans un mois donné cumulé sur la période $1972 / 82$, on trouve que les fractions d'insolation $\frac{S S}{S S_{\mathrm{o}}} \geqslant S_{\mathrm{g}}$ peuvent être classées en suites ininterrompues d'états $\Phi_{1}$. Ces dernières qui ne comportent que des transitions $\Phi_{1} \rightarrow \Phi_{1}$, sont par définition des séquences « $\Phi_{1} »$. De même, les valeurs $\frac{S S}{S S_{\mathrm{o}}}<S_{\mathrm{g}}$ induisent des chaînes d'états $\Phi_{0}$, appelées séquences « $\Phi_{0}$ ». Celles-ci ne comprennent que des étapes $\Phi_{0} \rightarrow \Phi_{0}$. L'analyse de ces données permet de dénombrer les séquences de climat de même durée et de dresser les histogrammes $N_{1}(k)$ et $N_{0}(k)$ qui donnent la fréquence des séquences respectives « $\Phi_{1}$ » et « $\Phi_{0}$ » en fonction de leur durée $k$, exprimée en nombre de jours.

D'après le modèle Markovien du premier ordre, la probabilité pour qu'une séquence de beau temps commençant par l'état $\phi_{1}$ ait une longueur de $k$ jours, est :

$$
\begin{aligned}
P\left(X_{2}=X_{3}=\cdots\right. & \left.=X_{k}=1, \quad X_{k+1}=0 / X_{1}=1\right)= \\
& =p_{10} p_{11}^{k-1}=A_{1} \exp \left(-a_{1} k\right)
\end{aligned}
$$

avec $A_{1}=p_{10} \exp \left(-\operatorname{Ln} p_{11}\right)$ et $a_{1}=-\operatorname{Ln} p_{11}$.

De même, la probabilité pour qu'une séquence de mauvais temps débutant par l'état $\phi_{0}$ dure $k$ jours, s'écrit :

$$
\begin{aligned}
P\left(X_{2}=X_{3}=\cdots\right. & \left.=X_{k}=0, \quad X_{k+1}=1 / X_{1}=0\right)= \\
& =p_{01} p_{00}^{k-1}=A_{0} \exp \left(-a_{0} k\right)
\end{aligned}
$$

avec $A_{0}=p_{01} \exp \left(-\operatorname{Ln} p_{00}\right)$ et $a_{0}=-\operatorname{Ln} p_{00}$.

L'analyse des données $S S / S S_{\mathrm{o}}$ en fonction du seuil montre que ce dernier présente une valeur particulière $S_{\mathrm{g}_{\mathrm{o}}}\left(\Phi_{1}\right)$ correspondant à une distribution Markovienne des points $\left\{k, N_{1}(k)\right\}$. Dans ce cas, ces points se répartissent selon une loi exponentielle, définie à une constante près par l'expression (2), et l'état $\Phi_{1}$ devient un état de beau temps $\phi_{1}$. De 
même, il existe une valeur remarquable $S_{\mathrm{g}_{0}}\left(\Phi_{0}\right)$ du seuil qui permet d'ajuster le nuage de points $\left\{k, N_{0}(k)\right\}$ à la loi de probabilité (3). Par suite, l'état $\Phi_{0}$ correspondant est un état de mauvais temps $\phi_{0}$. On voit donc que le seuil $S_{\mathrm{g}}$ est un paramètre essentiel car il permet d'ajuster le modèle Markovien aux données d'insolation.

2.3 TEST DE L'AJUSTEMENT. - L'ajustement est beaucoup plus aisé à réaliser quand les ordonnées sont à échelles logarithmiques. Aux données expérimentales $N_{1}(k)$ et $N_{0}(k)$, il correspond alors des quantités du type $y(k)=\operatorname{Ln} N(k)$ (en faisant abstraction des indices 1 et 0 ). D'autre part, en prenant le logarithme des probabilités (2) et (3), on a des droites de la forme :

$$
y_{\mathrm{o}}(k)=-a k+\operatorname{Ln} A \text {. }
$$

Les paramètres $a_{1}, A_{1}, a_{0}$ et $A_{0}$ peuvent être estimés par la méthode des moindres carrés, en minimisant la somme :

$$
S=\sum_{k} \frac{\left\{y(k)-y_{\mathrm{o}}(k)\right\}^{2}}{\sigma_{k}^{2}}
$$

où $\sigma_{k}$ est l'écart-type de la série des séquences $N(k)$.

Le calcul montre que l'expression (5) suit une loi de $\chi^{2}$, du type $\chi_{n-l-1}^{2}$ (où $n$ est le nombre d'observations et $l$ est le nombre de degrés de liaison) [9]. On en déduit que les paramètres d'ajustement peuvent être exprimés en fonction de la moyenne mensuelle, cumulée sur onze ans, des grandeurs $k, k^{2}, \operatorname{Ln} N(k)$ et $k . \operatorname{Ln} N(k)$. D'où :

$$
\begin{aligned}
a & =\frac{\bar{k} \cdot \overline{\operatorname{Ln} N(k)}-\overline{k \cdot \operatorname{Ln} N(k)}}{\overline{k^{2}}-\bar{k}^{2}} \\
\operatorname{Ln} A & =\frac{\overline{k^{2}} \cdot \overline{\operatorname{Ln} N(k)}-\bar{k} \cdot \overline{k \cdot \operatorname{Ln} N(k)}}{\overline{k^{2}}-\bar{k}^{2}} .
\end{aligned}
$$

Soient $\chi_{1}^{2}$, le test de l'ajustement des séquences de beau temps et $\chi_{0}^{2}$, celui des séquences de mauvais temps. Quand on minimise ces quantités, on obtient respectivement les seuils $S_{\mathrm{g}_{0}}\left(\chi_{1}^{2}\right)$ et $S_{\mathrm{g}_{\mathrm{o}}}\left(\chi_{0}^{2}\right)$. L'estimation de la taille de systèmes solaires requiert une valeur unique du seuil. Aussi, vaut-il mieux effectuer un test de $\chi^{2}$ global. Comme les événements (2) et (3) sont indépendants, il est possible d'additionner les valeurs $\chi_{1}^{2}$ et $\chi_{0}^{2}$. Le test de l'ajustement est alors réalisé avec la somme $\chi^{2}=\chi_{1}^{2}+\chi_{0}^{2}$. La somme (5) est minimum pour une valeur particulière $S_{\mathrm{g}_{0}}$ du seuil : celle qui donne la meilleure adaptation possible du modèle Markovien aux données d'ensoleillement.

\section{Traitement des données expérimentales.}

Les données d'insolation d'Alger ont été traitées par ordinateur afin de déterminer les valeurs mensuelles du seuil optimum $S_{\mathrm{g}_{0}}$, des différentes probabilités de transition et du coefficient de corrélation entre deux états consécutifs du climat.

3.1 Le SEUIL GLOBAL ANNUEL. - Un pas de calcul de 0,1 suffit pour explorer les différentes valeurs de seuil (un pas plus faible rendrait le traitement des données beaucoup plus complexe et le gain d'information serait minime). L'étude du seuil a donc été effectuée pour neuf valeurs de $S_{\mathrm{g}}$ allant de 0,1 à 0,9 . Les valeurs mensuelles de $S_{\mathrm{g}_{0}}$ qui en résultent, correspondent aux différents points de la courbe de la figure 2. On constate que celle-ci oscille autour de la moyenne annuelle du seuil global qui vaut $\bar{S}_{\mathrm{g}_{0}} \simeq 0,43$. Comme les conditions d'application $\mathrm{du}$ théorème central-limite sont pratiquement satisfaites, on a pour intervalle de confiance du seuil global de la population-mère : $\{0,34 ; 0,51\}$ (le risque de se tromper étant de 0,05 ).

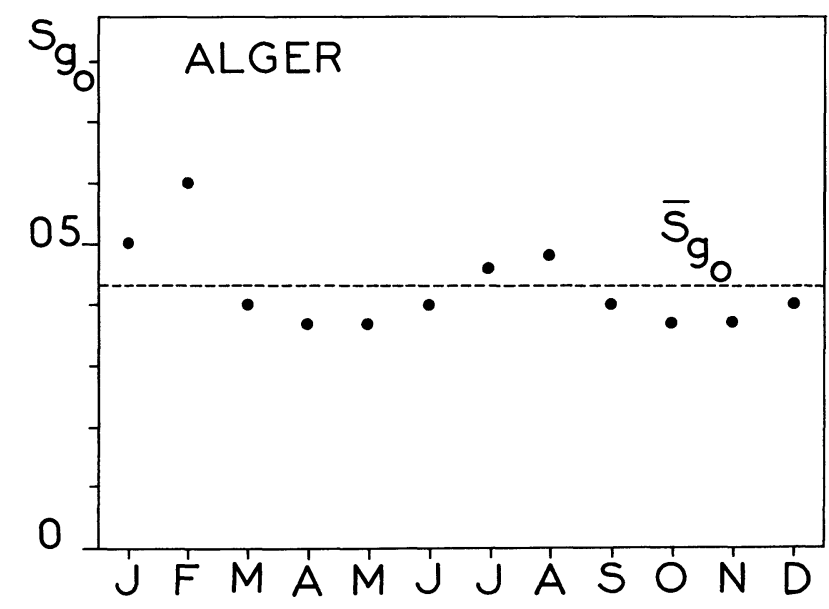

Fig. 2. - Variation des moyennes mensuelles du seuil de fraction d'insolation $S_{\mathrm{g}}$ au cours de l'année.

3.2 LeS PROBABILITÉS DE TRANSITION. - Les éléments de matrice $p_{i j}$ peuvent être évalués par recherche du maximum de vraisemblance. Considérons pour cela une séquence quelconque de climat qui se compose de $p$ transitions $\phi_{1} \rightarrow \phi_{1}, q$ transitions $\phi_{1} \rightarrow \phi_{0}, r$ transitions $\phi_{0} \rightarrow \phi_{1}$ et $s$ transitions $\phi_{0} \rightarrow \phi_{0}$. La probabilité de passage de l'état initial de la séquence vers son état final vaut

$$
\Pi=p_{11}^{p} \cdot p_{10}^{q} \cdot p_{01}^{r} \cdot p_{00}^{s} .
$$

Si on tient compte des relations (1e) et (1f) dans 
cette probabilité et si on fait $\frac{\partial \Pi}{\partial p_{11}}=\frac{\partial \Pi}{\partial p_{00}}=0$, il viendra :

$$
\hat{p}=\left[\begin{array}{ll}
\hat{p}_{11} & \hat{p}_{10} \\
\hat{p}_{01} & \hat{p}_{00}
\end{array}\right]=\left[\begin{array}{ll}
\frac{p}{p+q} & \frac{q}{p+q} \\
\frac{r}{r+s} & \frac{s}{r+s}
\end{array}\right] .
$$

$\hat{p}_{11}, \hat{p}_{10}, \hat{p}_{01}$ et $\hat{p}_{00}$ sont les valeurs respectives de l'estimation des paramètres $p_{11}, p_{10}, p_{01}$ et $p_{00}$. Les incertitudes commises sur ces estimations sont données par des écarts quadratiques de la forme :

$$
\sigma_{z}^{2}=\frac{(u+1)(v+1)}{(u+v+2)^{2}(u+v+3)}
$$

avec :

$$
\begin{aligned}
& u=p \text { et } v=q \text { si } \hat{Z}=\hat{p}_{11} \text { ou } \hat{p}_{10} \\
& u=r \text { et } v=s \text { si } \hat{Z}=\hat{p}_{01} \text { ou } \hat{p}_{00}
\end{aligned}
$$

Les probabilités $\bar{p}_{11}$ et $\bar{p}_{00}$ (et par suite, $\bar{p}_{10}$ et $\bar{p}_{01}$ ) sont estimées à partir des paramètres $p$, $q, r$ et $s$ qui ont été extraits des données d'insolation d'Alger mensuellement avec $\bar{S}_{\mathrm{g}_{\mathrm{n}}} \simeq 0,43$. D'où les diagrammes de la figure 3. Ceux-ci représentent les variations au cours de l'année des moyennes pondérées $\bar{p}_{11}$ et $\bar{p}_{00}$. Sur ces diagrammes, chaque valeur mensuelle de $\bar{p}_{11}$ ou de $\bar{p}_{00}$ a été liée à un segment vertical dont la longueur est proportionnelle à l'erreur d'estimation.

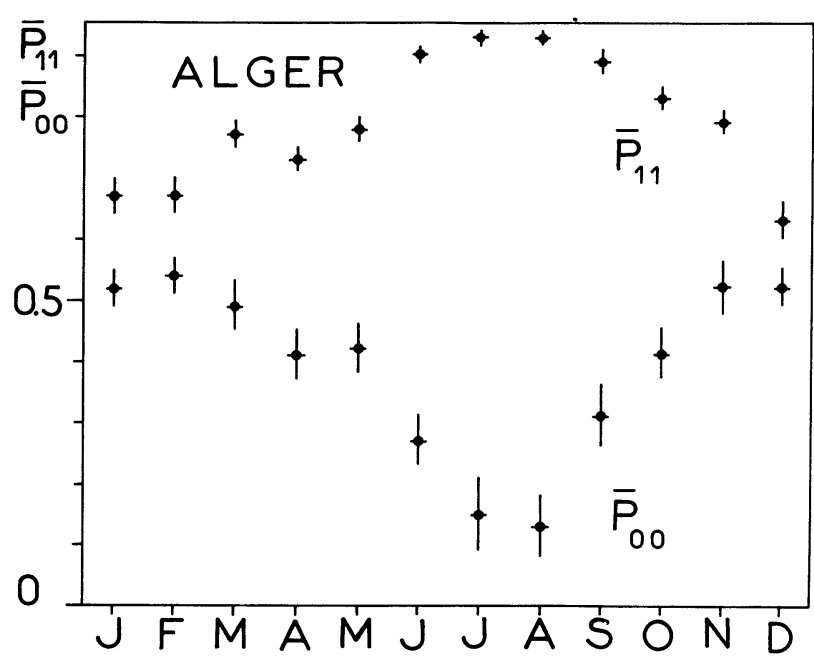

Fig. 3. - Evolution annuelle des moyennes pondérées (par l'erreur d'estimation) des probabilités de transition $p_{11}$ et $p_{00}$.

3.3 LE COEFFICIENT DE CORRÉlATION. - Considérons deux journées $j$ et $j+n$ séparées par un intervalle de $n$ jours et soient $X_{j}$ et $X_{j+n}$, les variables aléatoires associées aux deux journées. Le processus étant stationnaire, le calcul (développé dans l'appendice A) montre que le coefficient de corrélation linéaire est :

$$
C_{n}=\left(p_{11}-p_{01}\right)^{n}
$$

Le diagramme de la figure 4 indique comment évolue au cours de l'année le coefficient de corrélation $C_{1}$ de deux journées consécutives du climat de la zone d'Alger.

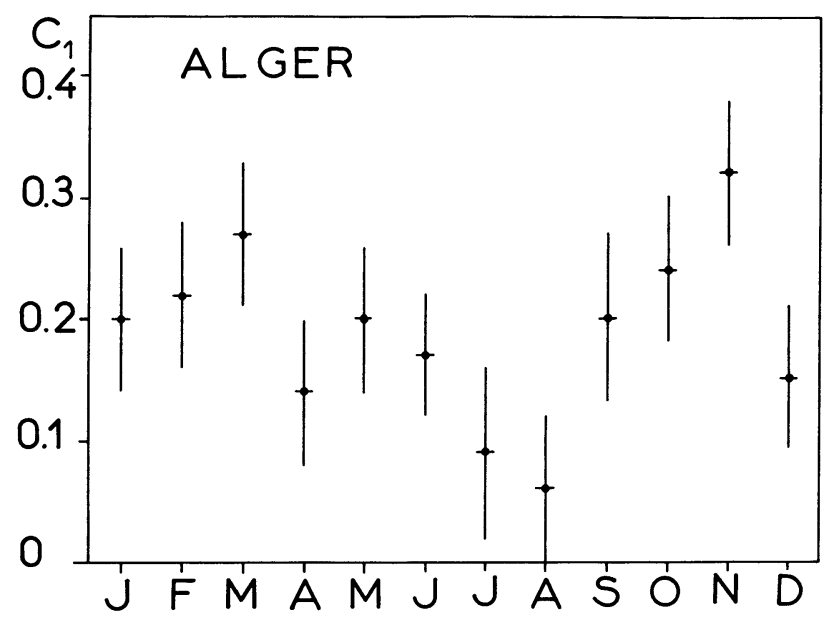

Fig. 4. - Variation au cours de l'année du coefficient de corrélation $C_{1}$.

\section{Analyse des résultats.}

D'après la figure 2 , le seuil passe par un maximum vers les mois de janvier et de février, puis vers les mois de juillet et d'août, où il est voisin de 0,5 . Il devient minimum et vaut pratiquement 0,35 au cours des périodes d'avril-mai et d'octobre-novembre. Deux arguments peuvent être invoqués pour expliquer l'allure de la courbe de la figure 2:

- Les valeurs de $S_{\mathrm{g}_{0}}$ ont été obtenues en dissociant les données $S S / S S_{\mathrm{o}}$ de façon à optimiser l'approximation Markovienne du premier ordre à deux états.

- Le nombre et le type de séquences " $\phi_{0}$ " et « $\phi_{1}$ » qui en découlent, diffèrent d'un mois à l'autre.

Les probabilités de transition décrites par la figure 3, varient aussi notablement avec les saisons et sont caractéristiques d'un climat méditerranéen. Ainsi la moyenne pondérée de la probabilité de beau temps qui est minimum en décembre et vaut 0,63 , devient maximum en juillet-août où elle est égale à 0,93 . D'autre part, les extrema de la moyenne pondérée de la probabilité de mauvais temps sont $\left(\bar{p}_{00}\right)_{\min }=0,13$ au mois d'août et $\left(\bar{p}_{00}\right)_{\max }=0,54$ en février. Les variations de $\bar{p}_{11}$ et de $\bar{p}_{00}$ ne font donc que traduire une amélioration générale du climat 
lors de son évolution de l'hiver à l'été, puis de sa dégradation vers l'hiver suivant.

La moyenne annuelle du coefficient de corrélation est égale à 0,18 (voir Fig. 4). Ce résultat est voisin des valeurs de $C_{1}$ publiées dans la littérature $[5,7$, 10]. Cependant, on constate sur la figure 4 que, malgré la valeur élevée des erreurs d'estimation, ce paramètre varie en fonction des saisons.

Afin d'apprécier la validité du modèle, une comparaison a été effectuée entre les probabilités de beau temps (ou de mauvais temps, selon le cas) déduites directement des données d'insolation et celles déterminées à l'aide du modèle Markovien, en régime permanent (voir Fig. 5). Ainsi, les probabilités $a$ priori $P\left(\phi_{1}\right)$ d'avoir un jour de beau temps et celles $P\left(\phi_{0}\right)$ d'avoir un jour de mauvais temps ont été calculées pour chaque mois de l'année. Sur les diagrammes de la figure 5 , elles correspondent aux différents points repérés par des croix. On en déduit qu'en moyenne au cours d'une année, nous avons 263 jours de beau temps et 102 jours de mauvais temps : ce qui reflète assez bien le type de climat de la région d'Alger. Par ailleurs, si on considère le régime permanent, la probabilité d'aboutir à un état de beau temps est $P\left(X_{j}=1\right)=p_{01} /\left(p_{01}+p_{10}\right)$. De même, la probabilité d'avoir du mauvais temps est $P\left(X_{j}=0\right)=p_{10} /\left(p_{01}+p_{10}\right)$. Ces expressions ont été obtenues par résolution de l'équation :

$$
\begin{aligned}
{\left[\begin{array}{ll}
P\left(X_{j}=1\right) & P\left(X_{j}=0\right) \\
P\left(X_{j}=1\right) & P\left(X_{j}=0\right)
\end{array}\right]\left[\begin{array}{ll}
p_{11} & p_{10} \\
p_{01} & p_{00}
\end{array}\right]=} \\
\quad=\left[\begin{array}{ll}
P\left(X_{j}=1\right) & P\left(X_{j}=0\right) \\
P\left(X_{j}=1\right) & P\left(X_{j}=0\right)
\end{array}\right] .
\end{aligned}
$$

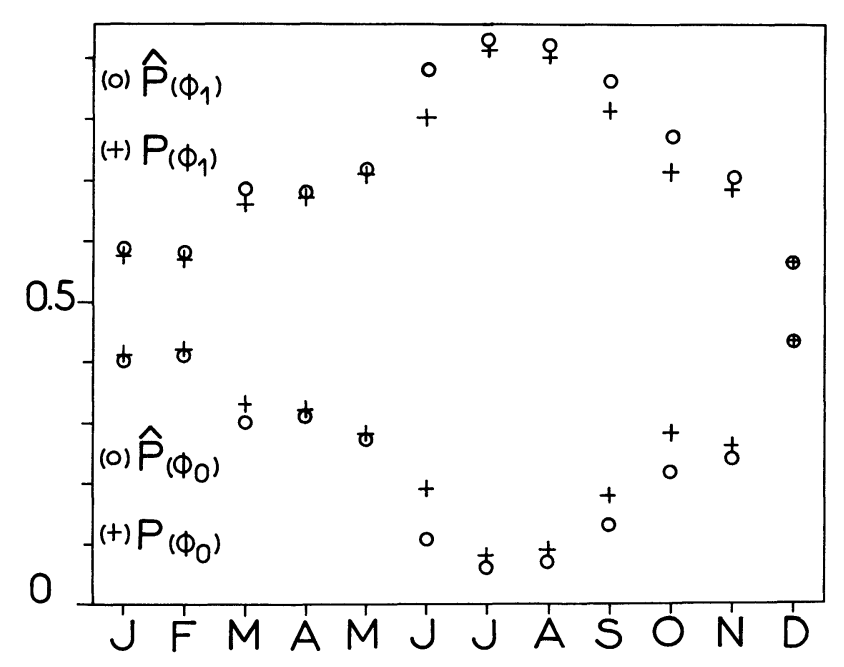

Fig. 5. - Comparaison des probabilités de beau temps $\hat{P}\left(\phi_{1}\right)$ et de mauvais temps $\hat{P}\left(\phi_{0}\right)$, déduites du modèle Markovien aux probabilités a priori correspondantes $P\left(\phi_{1}\right)$ et $P\left(\phi_{0}\right)$, résultant de l'observation. (Les cercles $O$ repèrent les valeurs mensuelles de $\hat{P}\left(\phi_{1}\right)$ et $\hat{P}\left(\phi_{0}\right)$. Les croix + correspondent aux probabilités $P\left(\phi_{1}\right)$ et $P\left(\phi_{0}\right)$.)
Après estimation par maximum de vraisemblance des paramètres $p_{i j}$, on obtient les valeurs mensuelles des probabilités $\hat{P}\left(\phi_{1}\right)=\hat{P}\left(X_{j}=1\right)$ et $\hat{P}\left(\phi_{0}\right)=$ $\hat{P}\left(X_{j}=0\right)$, repérées par des petits cercles sur la figure 5 .

La distribution des valeurs mensuelles de $\hat{P}\left(\phi_{1}\right)$ et de $P\left(\phi_{1}\right)$ d'une part et celle de $\hat{P}\left(\phi_{0}\right)$ et de $P\left(\phi_{0}\right)$ d'autre part montrent qu'il y a un bon accord entre les résultats du modèle et de l'observation.

\section{Conclusion.}

La méthode qui vient d'être décrite, permet de déterminer le seuil de fraction d'insolation alors que jusqu'à maintenant, celui-ci semble avoir été choisi empiriquement dans les différentes études de modélisation du rayonnement solaire [2,7].

L'ajustement des données d'insolation d'Alger par un modèle Markovien du premier ordre à deux états confirme bien le caractère stochastique du rayonnement solaire. Pour une meilleure prédiction de l'évolution de l'ensoleillement pendant l'année, il serait intéressant d'étendre cette étude à différentes régions et de dresser des cartes mensuelles des paramètres du modèle.

Nos résultats. laissent entrevoir la possibilité d'appliquer ce modèle à l'optimisation de la taille de systèmes solaires. Mais, si on doit faire intervenir le flux global d'irradiation solaire - comme c'est le cas pour le dimensionnement de convertisseurs photovoltaïques à orientation fixe - il faudrait adapter le modèle en conséquence.

\section{Remerciements.}

Nous remercions bien vivement le Dr R. Lestienne pour ses précieux conseils et l'Office National de la Météorologie pour avoir mis gracieusement à notre disposition les données d'ensoleillement d'Alger.

\section{Appendice A.}

Cet appendice est un exposé de la méthode d'évaluation de la corrélation qui existe entre le type de temps observé pour deux journées séparées par un intervalle de $n$ jours.

Le coefficient de corrélation linéaire est par définition :

$$
C_{n}=\frac{\operatorname{Cov}\left(X_{j}, X_{j+n}\right)}{\sigma_{X_{j}} \sigma_{X_{j+n}}} .
$$

Le processus Markovien considéré étant stationnaire, on a : $E\left[X_{j+n}\right]=E\left[X_{j}\right]$ et $\sigma_{X_{j+n}}=\sigma_{X_{j}}$. Dans ce cas, le coefficient de corrélation devient :

$$
C_{n}=\frac{E\left[X_{j} \cdot X_{j+n}\right]-\left(E\left[X_{j}\right]\right)^{2}}{\sigma_{X_{j}}^{2}}
$$


avec :

$$
\sigma_{X_{j}}^{2}=E\left[X_{j}^{2}\right]-\left(E\left[X_{j}\right]\right)^{2}
$$

La loi de probabilité de la variable aléatoire $X_{j}$ est l'ensemble des deux couples $\left\{0, P\left(X_{j}=0\right)\right\}$ et $\left\{1, P\left(X_{j}=1\right)\right\}$. Par suite, on aura :

$$
E\left[X_{j}\right]=P\left(X_{j}=1\right)=\frac{p_{01}}{p_{01}+p_{10}} .
$$

D'autre part, on a par définition :

$$
E\left[X_{j} \cdot X_{j+n}\right]=\sum \sum X_{j} X_{j+n} P\left(X_{j} \cap X_{j+n}\right) .
$$

Cette expression se réduit à :

$$
E\left[X_{j} \cdot X_{j+n}\right]=P\left(X_{j}=1 \cap X_{j+n}=1\right) .
$$

Or :

$$
\begin{aligned}
& P\left(X_{j}=1 \cap X_{j+n}=1\right)= \\
& \quad=P\left(X_{j}=1\right) \cdot P\left(X_{j+n}=1 / X_{j}=1\right)=\frac{p_{01} p_{11}^{(n)}}{p_{01}+p_{10}} .
\end{aligned}
$$

$p_{11}^{(n)}$ étant déduit de l'équation $p^{n-1} \cdot p=p^{n}$, on obtient la relation de réccurence :

$$
p_{11}^{(n)}=p_{11} p_{11}^{(n-1)}+p_{01} p_{10}^{(n-1)}
$$

avec :

$$
p_{10}^{(n-1)}=1-p_{11}^{(n-1)} .
$$

Par suite, on peut écrire que :

$$
\begin{aligned}
p_{11}^{(n)}=p_{11}\left(p_{11}-p_{01}\right)^{n-1} & + \\
& +\sum_{m=0}^{n-2} p_{01}\left(p_{11}-p_{01}\right)^{m} .
\end{aligned}
$$

Il vient, après calcul :

$E\left[X_{j} \cdot X_{j+n}\right]=\frac{p_{01}\left\{p_{01}+p_{10}\left(p_{11}-p_{01}\right)^{n}\right\}}{\left(p_{01}+p_{10}\right)^{2}}$.

Par ailleurs, si on tient compte du fait que $E\left[X_{j}^{2}\right]=E\left[X_{j}\right]$, l'expression (A.3) devient :

$$
\sigma_{X_{j}}^{2}=E\left[X_{j}\right]\left(1-E\left[X_{j}\right]\right)=\frac{p_{01} p_{10}}{\left(p_{01}+p_{10}\right)^{2}} .
$$

En combinant les relations (A.2), (A.4), (A.11) et (A.12), on trouve :

$$
C_{n}=\left(p_{11}-p_{01}\right)^{n}
$$

\section{Bibliographie}

[1] Boch, G., Bolleau, E. et Benard, C., Revue Phys. Appl. 16 (1981) 237.

Astier, R., Duhamel, C. et Benard, C., Revue Phys. Appl. 18 (1983) 29.

Benard, C., Boileau, E. et Guerrier, B., Revue Phys. Appl. 20 (1985) 845.

Bishnoi, C. P. and Saxena, K. K., Dev. Atm. Sci. 13 (1980) 197.

Madsen, H., Splid, H. and Thyregod, P., J. Clim. Appl. Meteo. 24 (1985) 629.

[2] Mejon, M. J., Bois, Ph. et Lestienne, R., Revue Phys. Appl. 15 (1980) 113.

[3] Box, G. E. P. and Jenkins, G. M., Time Series Analysis, Forecasting and Control (ed. Holden Day, San Francisco) 1976.

[4] Desvignes, F., Acta Electron. 5 (1961) 315. Buresh, M., Photovoltaic Energy Systems, Design and Installation (ed. MacGraw Hill, New York) 1983.

Barra, L., Catalanotti, S., Fontana, F. and LAVORANTE, F., Solar Energy 33 (1984) 509.

[5] Brinkworth, B. J., Solar Energy 19 (1977) 343.

[6] Amato, U., Andretta, A., Bartoli, B., Coluzzi, B., Cuomo, V. and Serio C., Il Nuovo Cimento, 8C (1985) 248.

[7] Lestienne, R., La Météorologie 12 (1978) 53. Lestienne, R., Revue Phys. Appl. 14 (1979) 139.

[8] CARTON, D., Processus aléatoires utilisés en recherche opérationnelle (éd. Masson, Paris) 1975.

[9] SAPORTA, G., Théories et méthodes de la statistique (éd. Technip, Paris) 1978.

[10] Bartoli, B., Coluzzi, B., Cuomo, V., FranCESCA, M. and Serio, C., Il Nuovo Cimento 4C (1981) 113. 